$\xi=-1$

\title{
Application of zero phase wavelet on synthetic seismic signals with noise
}

\author{
Sai Srinivas Goli ${ }^{1 *}$, Sireesha Papanaboyina ${ }^{2}$, Satya Ramesh Kanchumarthi ${ }^{3}$, Koteswara Rao Sanagapallea ${ }^{4}$
}

${ }^{1}$ Student KLEF

${ }^{2}$ Student KLEF

${ }^{3}$ Professor KLEF

${ }^{4}$ Professor KLEF

*Email: gsaisrinivas8@gmail.com

\begin{abstract}
Detection of time of occurrences of different phases and frequencies is of highest importance in seismic reflected signals. Seismic reflection analysis gives us accurate information about the event detection, source acquisition of triggered seismic data and its mechanisms. In the present work an attempt is made to generate a synthetic seismic with noise generally present in the seismograph using. The synthetic seismic signal is extracted by zero phase wavelet. Crews software is used in this extraction. The zero phase wavelet could efficiently extract the seismic signal present in the reflected wave.
\end{abstract}

Keywords: Seismic reflected signal; Synthetic seismic signal; Zero phase wavelet

\section{Introduction}

Seismic reflected signals are generally contaminated with various types of noises. The main aim of analyzing the seismic reflected signals is to interpret the regional and short period seismograms. The onset of various seismic arrivals leads to the detection of various sources and their mechanisms [1]. This also helps in the segmentation of seismographs into different wave groups. The discrimination on different types of event is done basing on onset time of the waves. The great variability in the spectra and amplitude of the signals provides robustness in achieving these goals. These signal generally waves are divided as body waves, surface waves. Generally Body waves can transverse through interior portion of the earth [2]. Whereas the surface waves will move along earth surface. Body waves can again spilt up into a primary $\mathrm{P}$-wave and a secondary $\mathrm{S}$-wave. It is reported that velocity of the $\mathrm{P}$-wave increase in rocks, liquids or through the liquid surface of the earth whereas the $\mathrm{S}$-waves is the second fastest wave which cannot move through a liquid medium [3].

The seismograph data is non-stationary in nature. It consists of different signals of different phases and frequencies. It is a challenging task to extract the original seismic signal hidden in this noise. Non-stationary signals are those which do not have constant mean and variance. Due to this property it is difficult to extract these signals using traditional signal processing techniques such as Fourier transforms, STFT etc. Wavelet transforms, an advanced signal processing techniques by which the information of time of occurrences of different frequencies can be extracted. Wavelets are signals having finite length. They are fast decaying oscillations which help in the detection of discontinuities and transients in the given data. Wavelet analysis eliminates the signal leakages and phase shifting of signals [4]. These functions resolve low frequencies in time domain and high frequencies in frequency domain.
These are highly suitable for signals spanning a wider spectrum such as seismic reflected signals.

The condition of zero phases makes the interpretation of seismic data accurate. So processing of data in these days makes effort to deliver every required data in condition of zero phases. Zero phase wavelet is mainly helpful to get result from the wavelet symmetry [5].

In the present work Crews matlab package is used for generating synthetic seismic reflected signals. The original seismic signal is extracted by using zero phase wavelet. Containing a large data concerning to geophysical routines. This software is subset of crews library. In this paper we have verified each module component for consistency and accuracy.

\section{Methodology}

Convolution is the process stationary linear filter to a signal and impulse response filter. We are using impulse response filter to the signal.

$$
p(s)=e_{0} h\left(s-s_{0}\right)+e_{1} h\left(s-s_{1}\right)
$$

Where $\mathrm{p}(\mathrm{s})$ is the filter output and $\mathrm{e}_{0}, \mathrm{e}_{1}$ are magnitudes and $\mathrm{s}_{0}, \mathrm{~s}_{1}$ are time.

$$
p(s)=\sum_{d=1}^{b} e_{d} h\left(s-s_{d}\right)
$$

Both the above equations are illustration of combining expressions, though even equations think too much about the nature of the input to be taken generally. The element is the development of a superposition of scaled-and-postponed duplicates of a solitary capacity $\mathrm{h}(\mathrm{s})$. Here, in examination for the over equation, a mix need displaced those sigma, the mix variable $\Phi$ need taken the 
spot of the sigma list $\mathrm{b}_{2 \times 2}$ and the sequence of impulses has become the straight function $\mathrm{e}(\Phi)[6]$.

$$
p(s)=\int_{-\infty}^{\infty} e(\varphi) h(s-\varphi) d \varphi
$$

The vital activity of equation above is symbolized abstractly as q $=\mathrm{e} \bullet \mathrm{h}$, then it is rudimentary to show that $\mathrm{e} \bullet \mathrm{h}=\mathrm{h} \bullet \mathrm{e}$. This is achieved by the change of variables $\varphi=S-\varphi$ that gives [7]

$$
\begin{aligned}
p(s) & =\int_{-v}^{v} e(\varphi) h(s-\varphi) \mathrm{d} \varphi \\
& =\int_{v}^{-v} e\left(s-\varphi^{\prime}\right) h\left(\varphi^{\prime}\right) \mathrm{d} \varphi^{\prime} \\
& =\int_{-v}^{v} e\left(s-\varphi^{\prime}\right) h\left(\varphi^{\prime}\right) \mathrm{d} \varphi^{\prime} \\
\mathrm{q}_{\mathrm{w}}=\Delta \mathrm{s} & \sum_{d=0}^{B-1} e_{d} h_{w-d}
\end{aligned}
$$

Where the standard $\Delta \mathrm{s}$ had took out of sigma. In most executions of digital signal theory, $\Delta \mathrm{s}$ is steady for whole application and it is standard to write equations with $\Delta \mathrm{s}$ kept as 1 . So, most of the discrete convolutions will be written as

$\mathrm{q}$ w $=\sum_{d=0}^{B-1} e_{d} h_{w-d}$

Normally those essential analytics of the result from claiming two nonstop works camwood make approximated over a workstation as a matrix-vector item. Those convolution essential analytics gives a critical illustration. Consider the discrete convolution formula

$\mathrm{q}_{\mathrm{w}}=\sum_{d=0}^{B-1} e_{w-d} h_{d}$

Consider the Fourier transform of the time-shifted signal y $(s+\Delta s)$

$Y(h)=\int_{-\infty}^{\infty} y(s+\Delta s) e^{i 2 \pi h s} d s$

and make the change to the variables $\varphi=s+\Delta s$ and look it with the normal equation for the multiplication of a matrix, $D_{w d}$ with a vector $r_{d}$ that is

$$
T_{w}=\sum_{b} D_{w d} r_{d}
$$

Convolution will be executed by characterizing a matrix $E_{w d}=e_{w-d}$ written as a matrix equation and the above equation becomes

$$
\left[\begin{array}{c}
q_{0} \\
q_{1} \\
q_{2} \\
\cdot \\
\cdot \\
\cdot \\
q_{B-1}
\end{array}\right]=\left[\begin{array}{ccccccc}
e_{0} & e_{-1} & e_{-2} & \cdot & \cdot & \cdot & e_{-B+1} \\
e_{1} & e_{0} & e_{-1} & \cdot & \cdot & \cdot & e_{-B+2} \\
e_{2} & e_{1} & e_{0} & \cdot & \cdot & \cdot & e_{-B+3} \\
\cdot & \cdot & \cdot & \cdot & \cdot & \cdot & \cdot \\
\cdot & \cdot & \cdot & \cdot & \cdot & \cdot & \cdot \\
\cdot & \cdot & \cdot & \cdot & \cdot & \cdot & \cdot \\
e_{B-1} & e_{B-2} & e_{B-3} & \cdot & \cdot & \cdot & e_{0}
\end{array}\right] \cdot\left[\begin{array}{c}
h_{0} \\
h_{1} \\
h_{2} \\
\cdot \\
\cdot \\
\cdot \\
h_{B-1}
\end{array}\right]
$$

One approach to see the matrix-vector multiplication is called matrix multiplication by horizontal lines. In this process, each row of $\mathrm{E}$ multiplies $\mathrm{h}$ as a vector dot product. That is, those comparing components need aid increased and the coming about items summed will a scalar that gets the comparing component for $\mathrm{g}$. This can be written as set of equations [7] $\mathrm{q}_{0}=\mathrm{e}_{0} \mathrm{~h}_{0}+\mathrm{e}_{-1} \mathrm{~h}_{1}+\mathrm{e}_{-2} \mathrm{~h}_{2}+\ldots \mathrm{e}_{-\mathrm{Z}+1} \mathrm{~h}_{\mathrm{Z}-1}$

$\mathrm{q}_{1}=\mathrm{e}_{1} \mathrm{~h}_{0}+\mathrm{e}_{0} \mathrm{~h}_{1}+\mathrm{e}_{-1} \mathrm{~h}_{2}+\ldots \mathrm{e}_{-\mathrm{Z}+2} \mathrm{~h}_{\mathrm{Z}-1}$

$\mathrm{q}_{2}=\mathrm{e}_{2} \mathrm{~h}_{0}+\mathrm{e}_{1} \mathrm{~h}_{1}+\mathrm{e}_{0} \mathrm{~h}_{2}+\ldots \mathrm{e}_{-\mathrm{Z}+3} \mathrm{~h}_{\mathrm{Z}-1}$

$\ldots=\ldots$

$\mathrm{qZ}^{-1}=\mathrm{eZ}-1 \mathrm{~h}_{0}+\mathrm{eZ}^{-2} \mathrm{~h}_{1}+\mathrm{eZ}^{-3} \mathrm{~h}_{2}+\ldots . \mathrm{e}_{0} \mathrm{hZ}^{-1}$

The picture of matrix product shows how each out sample of $q$ is created as a straight superposition of the inputs. An option, but similarly legitimate point of view comes from matrix product by vertical lines. Review of the expressions tells that each term of $h$ multiplies a corresponding column of $\mathrm{E}$ [8].

$$
\begin{aligned}
\mathrm{Y}(\mathrm{h}) & =\int_{-v}^{v} y(\varphi) e^{i 2 \pi h(\varphi-\Delta s)} d \varphi \\
& =e^{-i 2 \pi h \Delta s} \int_{-v}^{v} y(\varphi) e^{i 2 \pi h \varphi} d \varphi \\
& =e^{-i 2 \pi h \Delta s} \mathrm{Y}(\mathrm{h})
\end{aligned}
$$

\section{Results}

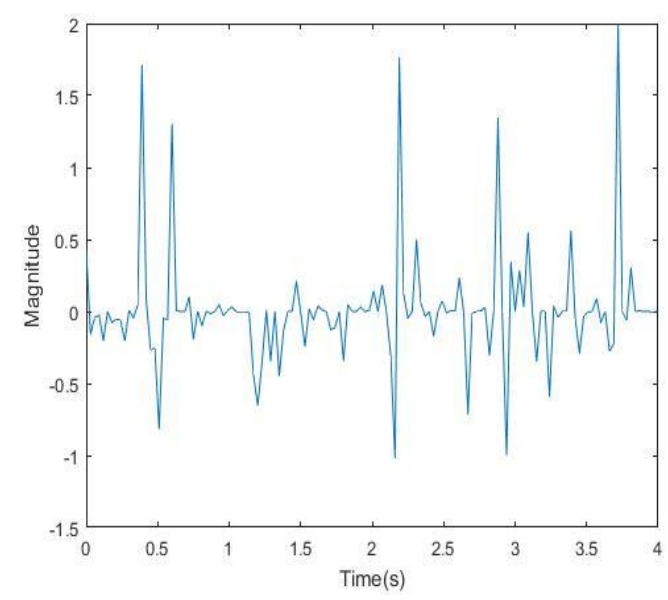

Fig. 1: Synthetic pseudo random reflected signal

Fig. 1 is a synthetic pseudo random reflected signal with record length of 4 , sample rate of 0.03 and amplitude of 2 .

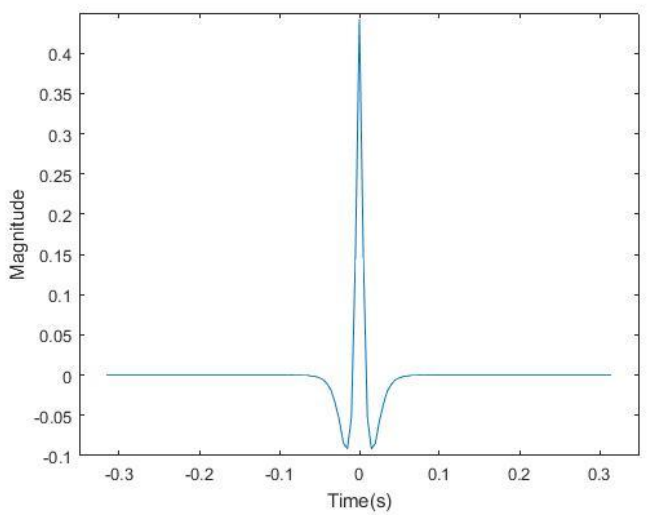

Fig. 2: Zero phase wavelet 
Fig. 2 is a zero phase wavelet with a temporal sample rate (dt) of 0.005 , dominant frequency (fdom) of $20 \mathrm{~Hz}$ and length of wave (tlength) $127 *$ temporal sample rate $(0.635)$.

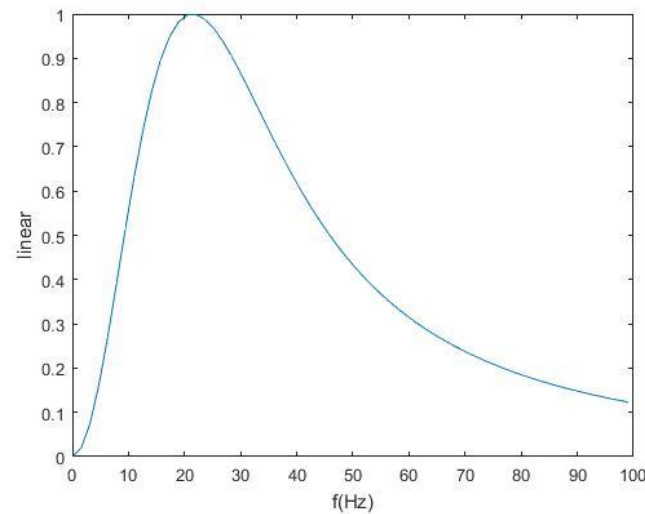

Fig. 3: Amplitude spectrum on a linear scale

Fig.3 describes about the spectrum of amplitude on a linear scale with frequency on $\mathrm{X}$-axis and amplitude (linear scale) on $\mathrm{Y}$-axis.

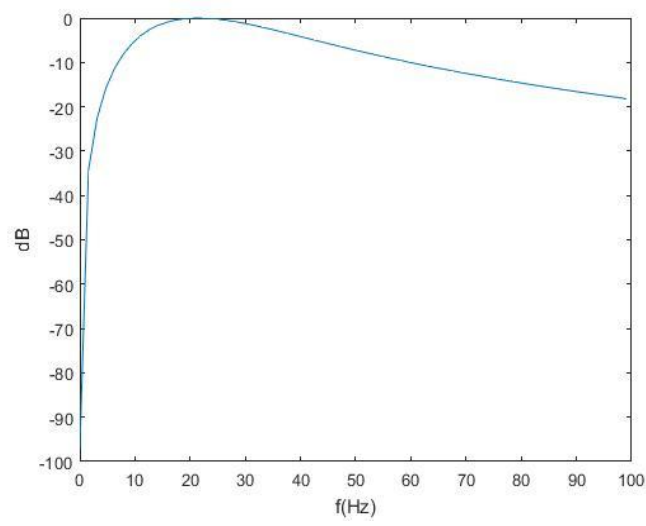

Fig. 4: Amplitude spectrum on a decibel scale

Fig.4 indicates the amplitude spectrum on a decibel (dB) scale with frequency on $\mathrm{X}$-axis and amplitude on $\mathrm{Y}$-axis.

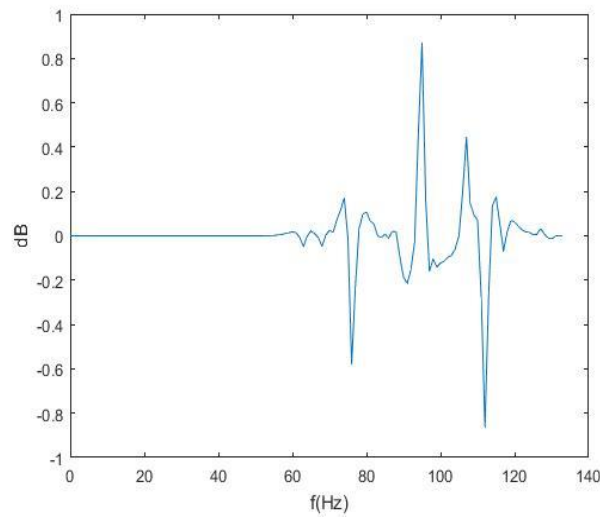

Fig. 5: Synthetic seismogram using zero phase wavelet

Fig.5 describes the synthetic seismogram using a zero phase wavelet by convolving the original wavelet with the reflected wavelet with frequency on $\mathrm{X}$-axis and amplitude on $\mathrm{Y}$-axis with limits ranging from 0 to length of the convolved signal $-1 / 2$.

\section{Conclusion}

In this paper, It is observed that the synthetic seismic signal, Extracted from the zero phase wavelet is analyzed, It seems that the method used by us is better when compared to other wavelet transforms for the detection of earthquakes.

\section{References}

[1] Aim and Scope of the IASPEI New Manual of Seismological Observatory Practice

(P. Bormann) DOI:10.2312/GFZ.NMSOP_r1_ch1.

[2] Seismic Arrays (J. Schweitzer, J. Fyen, S. Mykkeltveit and T. Kvaerna) DOI: $10.2312 /$ GFZ.NMSOP_r1_ch9.

[3] Rosenburg. Real-Time Ground-Motion Analysis: Distinguishing P and S Arrivals in a Noisy Environment. Bulletin of the Seismological Society of America, 100(3):1252-1262, June 2010.

[4] K. M. Taylor, M. J. Procopio, C. J. Young, and F. G. Meyer. Estimation of arrival times from seismic waves: a manifold based approach. Geophysical Journal International, 185:435-452, 2011.

[5] P. shearer. introduction to seismology. cambridge university press, 2006.

[6] united states geographical survey. earthquake hazards program: http://earthquake.usgs.gov/earthquakes/eqarchives/year/eqstats. php, april 2012.

[7] Learning from Manifold-Valued Data: An Application to Seismic Signal Processing by Juan Ramirez Jr.

[8] Seismic Signals and Noise(P. Bormann) DOI: $10.2312 /$ GFZ.NMSOP r1 ch4.

[9] Ground-Based Nuclear Explosion Monitoring Technologies A Model based signal processing approach to seismic monitoring. Arthur J. Rodgers, David B. Harris, and Michael E. Pasyanos. 